\title{
BMJ Open Multicentre prospective cohort study of unmet supportive care needs among patients with breast cancer throughout their cancer treatment trajectory in Penang: a PenBCNeeds Study protocol
}

To cite: Mohd Shariff N, Azman N, Hami R, et al. Multicentre prospective cohort study of unmet supportive care needs among patients with breast cancer throughout their cancer treatment trajectory in Penang: a PenBCNeeds Study protocol. BMJ Open 2021;11:e044746. doi:10.1136/ bmjopen-2020-044746

- Prepublication history is published online only. To view please visit the journal online (http://dx.doi.org/10.1136/ bmjopen-2020-044746).

Received 11 September 2020 Revised 14 January 2021 Accepted 23 February 2021

\section{Check for updates}

(c) Author(s) (or their employer(s)) 2021. Re-use permitted under CC BY-NC. No commercial re-use. See rights and permissions. Published by BMJ.

${ }^{1}$ Advanced Medical and Dental Institute, Universiti Sains

Malaysia, Kepala Batas, Penang, Malaysia

${ }^{2}$ Biostatistics and Bioinformatics Unit, Research and Networking Department, Advanced Medical and Dental Institute, Universiti Sains Malaysia, Minden, Penang, Malaysia

Correspondence to Dr Noorsuzana Mohd Shariff; suzanashariff@usm.my

\section{ABSTRACT}

Introduction Proper assessment of unmet supportive care needs of patients with breast cancer and its influencing factors at different treatment intervals will improve the rehabilitation of patients with breast cancer. Therefore, this study aims to determine the prevalence of unmet supportive care needs, changes of needs over time and associated factors during the treatment period. Methods and analysis This multicentre, prospective cohort study will be conducted in three governmental hospitals and one tertiary cancer institute in Penang, Malaysia. Adult women diagnosed with primary or recurrent tumour, node, metastases stage I-IV breast cancer based on pathological biopsy will be eligible for this study. At least 281 samples are required for this study. Participants will undergo follow-up at three time intervals: T1 at breast cancer diagnosis; T2 at 3 months after diagnosis and T3 at 6 months after diagnosis. Patients will complete a set of questionnaires at each time. The primary outcome of this study includes the changes in supportive care needs over three time points, followed by the secondary outcome examining patients' characteristics, coping behaviours and positive psychological components as they affect changes in unmet supportive care needs over time.

Ethics and dissemination The study has received ethics approval from the Medical Research and Ethics Committee, Ministry of Health Malaysia (NMRR-19-26845809 IIR) and the Human Research Ethics Committee of Universiti Sains Malaysia (USM/JEPeM/17100443). The results of the prospective study will be submitted for publication in a peer-reviewed journal.

\section{INTRODUCTION}

\section{Background and rationale}

Breast cancer has long been a global noncommunicable disease threat. More than 2 million newly diagnosed patients with breast cancer were reported in 2018. ${ }^{1}$ Most of the patients with breast cancer were recorded in the Asia region, and Malaysia is among the countries contributing to the statistics.

\section{Strengths and limitations of this study}

This study is a prospective follow-up of newly diagnosed patients with breast cancer.

- Patients' supportive care needs will be assessed at three time points across their treatment trajectory: $\mathrm{T} 1$ at breast cancer diagnosis; $\mathrm{T} 2$ at 3 months after diagnosis and T3 at 6 months after diagnosis.

- This study will assess the effects of patients' sociodemographic characteristics, clinical status, treatment history, coping behaviour, social support and post-traumatic growth on the unmet supportive care needs of the patients.

- Two-way repeated-measures multivariate analysis of variance will be used to determine differences in multiple needs in all five domains over time and between-subject characteristics.

- This study may suffer from high attrition of study samples at second and third follow-up, but this may be countered with a proper data collection strategy and strong interpersonal skills among the team.

Breast cancer is the most common cancer, accounting for $34.1 \%$ of all cancer among women in Malaysia for the period 2012-2016. Alarmingly, $47.9 \%$ of these cancers were detected in their late stages (III and IV). ${ }^{2}$ Patients with cancer and cancer survivors face a wide range of problems throughout their treatment, which often persist in a chronic and long-term manner. ${ }^{3}{ }^{4}$ In-treatment complications are usually caused by the side effects of treatment, exposing patients to negative physical and mental symptoms and social function problems. ${ }^{56}$

As distinct from advanced technology for medical treatments, non-pharmacological approaches such as supportive care are essential to reduce patients' symptoms, and improve quality of life among patients and their families. ${ }^{78}$ Supportive care is defined 
as rendering essential services that satisfies the physical, psychological, social, informational and spiritual needs of patients with cancer over the entire illness trajectory. Crucial aspects in supportive care include social care, the need for information, psychological support and spiritual needs. These can all improve emotional and functional adjustment and symptoms and quality of life and may contribute to increased survival. ${ }^{9} 10$ Unmet needs are most prominent when gaps exist between a person's experience of services and the actual services required or desired. ${ }^{11}$ Understanding the prevalence of unmet needs of patients with breast cancer is crucial and clinically of prime importance throughout their continuum of survivorship to offer timely effective interventions. Moreover, it has been shown that increased unmet needs are associated with a range of adverse outcomes, including increases in self-reported anxiety and depression. ${ }^{12}$

The supportive care needs of patients with breast cancer have been explored with numerous study designs incorporating quantitative, qualitative or mixed-method approaches. ${ }^{13-19}$ Majority of studies adopt cross-sectional study design to assess unmet needs of patients with breast cancer at diverse cancer time points followed by several phenomenological qualitative interviews and longitudinal studies. ${ }^{4} 13-15$ 18 20-25 Qualitative studies exploring the unmet needs experienced by patients with breast cancer and cancer survivors have found several significant themes related to difficulty in finding information, physical discomfort, safety, emotions, financial and social support. ${ }^{15-17}$

A similar pattern of unmet needs has also been observed in many other cross-sectional studies in the past 10 years. Three domains reported as the most frequent unmet needs among patients with breast cancer are the need for a health system and information, ${ }^{4} 131518202425$ physical needs ${ }^{13-15} 18$ and psychological needs. ${ }^{14}$ 21-23 In general, unmet supportive care is more common among younger patients, later cancer stage, multiple breast cancer (multiplicity), human epidermal growth factor receptor 2 (HER2), treatment state, marital status, higher education, shorter time since diagnosis, employment and psychosocial status. ${ }^{1314192325}$ A Korean study showed that the older age group showed a higher level of recognition of physical symptom needs. In contrast, the unemployed group expressed more significant needs for information and education. Survivors with multiple breast cancers, indicated by two or more invasive tumours within the same breast quadrant and simultaneous existence of a tumour in the other breast, have greater needs in the domains of healthcare staff and physical symptom. ${ }^{182627}$

Efforts to observe changes of needs over a various predetermined time interval across the breast cancer treatment trajectory have been reported in a few previous studies. The assessment points vary from the diagnosis of breast cancer to the end of primary treatment, following treatment or post-treatment follow-up. ${ }^{28-31}$ A prospective longitudinal survey by Liao $e t a l^{28}$ assessed patients' needs at four intervals (at diagnosis (T1), 1 month (T2),
2 months (T3) and 3 months (T4) after diagnosis). They found that women had moderate-to-high levels of unmet needs, with the highest being in the health system and information domain at each time point. Interestingly, psychological, health system and information, and sexuality needs reduced over time, but not physical and daily living (PDL) needs, which increased significantly over time. ${ }^{28}$ Another longitudinal study was conducted among Chinese women with advanced breast cancer receiving initial chemotherapy. Each of the patients was assessed at baseline, then again at 6 weeks, 3 months, 6 months and 12 months post-baseline. Most women in the study showed stable low levels of health system, information and patient support (HSIPS) (78.9 \%); psychological (82.4 \%); PDL (83.7\%); and sexuality (97.4\%) supportive care needs. One in five and one in eight women showed high initial supportive care needs in HSIPS and psychological and PDL domains, respectively. ${ }^{32}$

The association of coping strategies, social support and post-traumatic growth (PTG) on supportive care needs has been demonstrated in a study among patients with haematological cancer. Higher perceptions of partners' negative dyadic coping are associated with higher supportive care needs for both patients and partners. ${ }^{33}$ However, the evidence of the effect of these factors on the changes in supportive care needs among patients with breast cancer is scarce. Limited evidence has shown a significant relationship between social support and unmet needs. ${ }^{21}$ Social support refers to physical, psychological and financial support available to an individual which is provided by a network of family, friends, neighbours and community members in times of need. ${ }^{34}$ Social support has four components: instrumental, informational, emotional and companionship support. Instrumental support includes the provision of financial assistance, services and goods support by the source of social support. Informational support includes provision of information, guidance and advice by the source of social support. Emotional support encompasses warm and affectionate support, such as love, protection, care, acceptance and trust as provided by the source of social support. Companionship support allows a sense of belonging to oneself, and spousal support is an example of companionship support and is of utmost importance to patients with cancer, as it enables positive reappraisal of the traumatic cancer experience..$^{35} \mathrm{~A}$ study of unmet social support needs among elderly cancer survivors indicated that two-thirds of cancer survivors had at least one unmet social support need. Commonly reported unmet social support needs among cancer survivors include emotional, physical, medical and informational social support needs. ${ }^{36}$

PTG is the development of positive psychological growth in response to a traumatic experience or lifethreatening event. PTG has five domains: appreciation of life, spiritual development, new possibility in life, greater interpersonal relationship and greater personal strength. PTG occurs when someone's initial pre-assumptive world is shattered by a traumatic event, but he or she is later 
able to make new meaning out of the traumatic event by incorporating the new trauma-related information into rebuilding a new assumptive world of self, others and surroundings. This is an enhancement of psychological functioning beyond the level experienced by the person prior to the traumatic event. ${ }^{37}$ PTG has been reported in patients with various cancer diagnoses, including patients with breast cancer. ${ }^{38}$ PTG has been shown to be associated with spousal support and coping mechanisms, such as positive reframing and religious coping in patients with cancer. ${ }^{39}$ To the best of our knowledge, there have been no studies to date which have investigated the association between PTG and unmet needs in patients with cancer. It is also not known whether PTG and unmet needs exhibit a bidirectional association in patients with cancer.

Prospective assessment of changes of unmet supportive care needs of patients with breast cancer and its influencing factors at different treatment intervals is in great demand to improve the quality of life and rehabilitation of patients with breast cancer. Assessing the needs also offers a direct measure of the patients' support preference and service gaps. In return, this will help clarify where actions and resource allocation are necessary for the healthcare setting in providing good quality patientcentred management to patients with breast cancer. ${ }^{32}$

\section{Specific aims}

\section{Aim 1}

To determine the prevalence of unmet supportive care needs experienced by patients with breast cancer at early diagnosis, 3 months after treatment and 6 months after treatment.

\section{Aim 2}

To determine significant changes in unmet supportive care needs of patients with breast cancer over the three points of time across five domains (psychological, PDL, sexuality, health system and information, and patient care and support).

\section{Aim 3}

To determine any associations between patients' sociodemographic characteristics, clinical status, treatment history, coping behaviour, social support and PTG, and changes in supportive care needs among patients with breast cancer.

\section{METHODS AND ANALYSIS \\ Study design}

This study will be conducted as a multicentre, prospective cohort study.

\section{Study area and study population}

This study will be conducted in three governmental hospitals and one tertiary cancer institute providing treatment to patients with breast cancer in Penang, Malaysia. Located in northwest peninsular Malaysia, Penang recorded the highest incidence of all cancer sites between
2012 and 2016. ${ }^{2}$ This study will involve newly diagnosed patients with breast cancer diagnosed between 1 July 2019 and 31 December 2020. The inclusion criteria include women with a confirmed diagnosis of breast cancer based on pathological biopsy and who: were aged 18 years and above; were diagnosed with primary or recurrent breast cancer less than 3 months from the histopathological examination date; had tumour, node, metastases stage IIV breast cancer; were Malaysian and provided consent to participate in the study. The exclusion criteria exclude women less than 18 years of age or with: no cancer of cancer in situ; secondary cancer; language or cognitive difficulties; or a diagnosis more than 3 months previously. Eligible women will be invited to participate in the project by a trained research assistant on their appointment day in the oncology clinic.

\section{Sample size estimation}

The sample size was calculated using the single proportion formula. ${ }^{40}$ The calculation was done based on the expected prevalence of unmet supportive care needs among patients with breast cancer $(79.0 \%)$ from the previous study conducted by Edib et al. ${ }^{10}$ The sample size was calculated with the requirements for significance level $(\alpha), 0.05$ and power $(1-\beta), 0.8$. Estimated $10 \%$ missing data have been added to the final figure. Therefore, a total of 281 samples are needed for this study.

\section{Sampling method and data collection procedure}

Participants will be recruited using convenience sampling of newly diagnosed patients with breast cancer attending oncology clinics in the four study sites. Convenience sampling was chosen due to its benefits in light of our limited time, budget and resources. ${ }^{41}$ With the help of nurses in the participating clinics, the research team will be updated with the names, contact details and date of next follow-up of newly diagnosed patients every month. From here, the research team will take charge of the other activities related to the study to minimise the effects of proxy involvement (for instance carer, healthcare providers) in study interviews on the patients' responses. ${ }^{42}{ }^{43}$ Each of the eligible patients will be contacted via telephone by the trained research assistant or the second author prior to their appointment day to make them aware of the study and the need for first face-to-face T1 interview. Patients will be excluded if they do not agree to participate. For those willing to participate, the research assistant will set an appointment for the first interview. The research assistant will approach eligible women on their appointment day during the waiting time. Each participant will be given explicit information about the study background, the purpose of the study and the data collection process. Informed consent will be taken before they enrolled in the study. Participants will be informed that there are three interviews involved in the study. Each participant will be given full autonomy to participate or withdraw from the study at any time without jeopardising their ongoing cancer treatment. Figure 1 depicted the flow of the data collection planned for this study. The patients' medical 


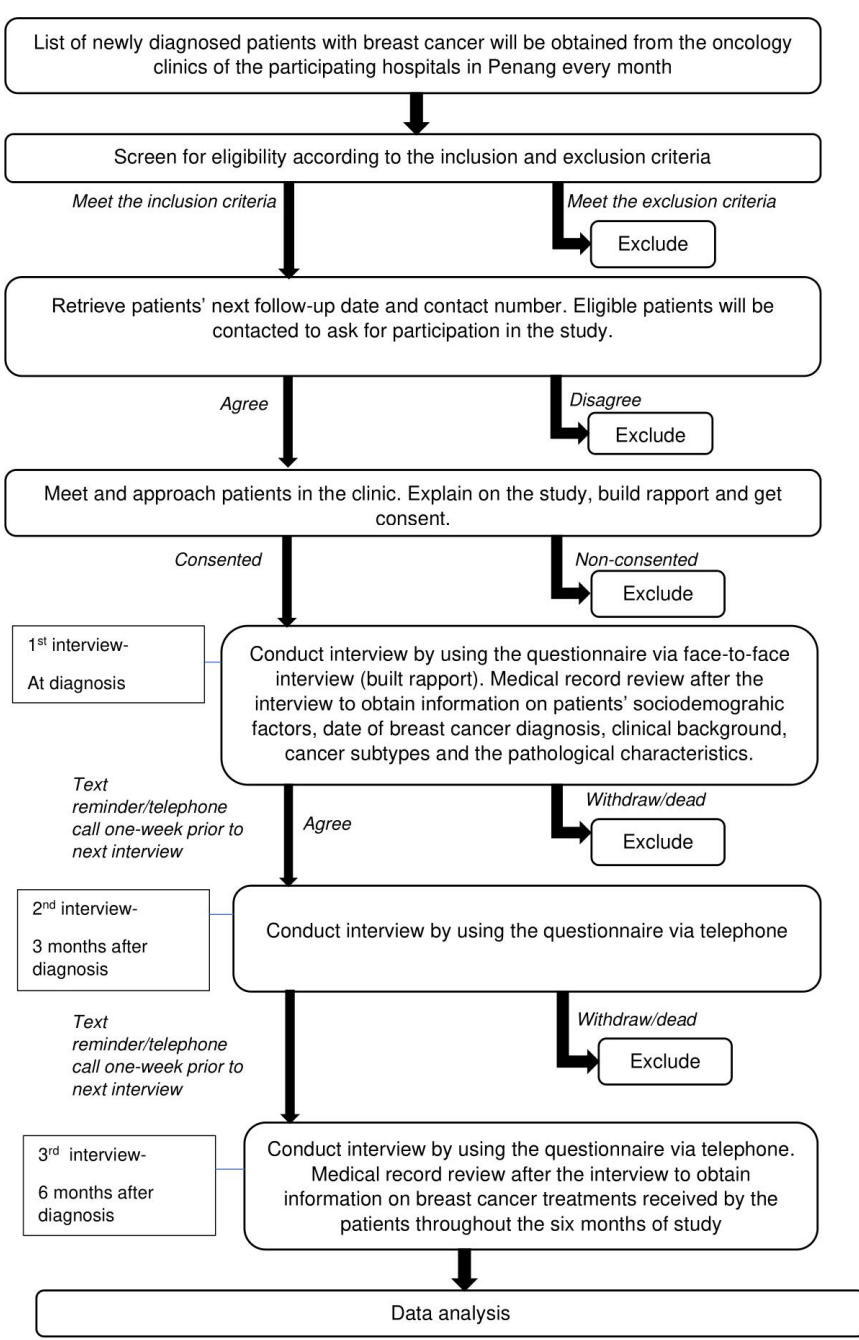

Figure 1 Data collection framework.

records will be reviewed twice throughout the study, first after the T1 interview to assess patients' sociodemographic factors, date of breast cancer diagnosis, clinical background, cancer subtypes and pathological characteristics. Next, medical records will be reassessed again by the trained research assistant and the second author after patients have completed the third interview to assess the breast cancer treatments received by the patients throughout the 6-month study. Patients who do not consent to this aspect of the study will be excluded from the study.

The 34-item Short-Form Supportive Care Needs Questionnaire has been shown to be valid and successfully used as either self-administered, face-to-face or telephone interviews previously. ${ }^{44} 45$ The survey will be conducted using face-toface and telephone interview modes of administration. The face-to face interview has shown to have several advantages over the self-administered mode of administration, as it requires only basic verbal and listening skills. No reading skills are required. This mode of administration has also shown to have high survey response and high questionnaire completion rates and low recall bias. Respondents prefer this mode of administration as compared with self-administered modes. ${ }^{46}$ The face-to-face interview method was selected for the first $\mathrm{T} 1$ interview as it will allow the interviewer to build rapport and strong trust with the patients, to reduce response rate discrepancies associated with the use of the telephone as a medium for conducting data collection in the other two follow-up interviews. ${ }^{47}$ Other researchers have demonstrated that when first personal contact is initiated, the differences between the response rates of the face-to-face interview and telephone interview methods disappear. ${ }^{49-51}$ Patients will become more easily familiar with the questions and the response scale in person, before being subsequently interviewed via telephone.

Data collection via telephone interview has two significant benefits, namely cost-effectiveness and time efficiency. ${ }^{4751} \mathrm{~A}$ follow-up interview by telephone reduces the direct cost of travelling to the clinic for interview for both the research team and the participants and takes less time overall. $^{52}$ At the end of the $\mathrm{T} 1$ interview, the reviewer will remind the patients of their next $\mathrm{T} 2$ interview via a telephone call after 3 months. A week before the set T2 interview date, patients will be sent a text reminder or a phone call by the research assistant. If rescheduling is needed, patients will be required to reply to the text/phone call. The research assistant will repeat these procedures for the T3 interviews to minimise attrition.

\section{Proposed statistical methods}

Data will be analysed using IBM SPSS Statistics Campus Edition V.27.0 for Windows (IBM Corp). Descriptive statistics will be used to summarise the characteristics of the subjects. Numerical data will be presented as either mean (SD) or median (IQR) based on normality distribution. Categorical data will be presented in terms of frequency and percentage. The prevalence of unmet supportive care needs experiencing by the patients with breast cancer at early diagnosis, 3 months after diagnosis, and 6 months after diagnosis for each of the five domains will be described in terms of frequency and percentage. Patients will be classified in a binary categorical group as having or not having unmet supportive care needs. Patients will be said to have unmet needs for each domain if they scored moderate-to-high needs in the respective domain. Next, for the purpose of assessing changes in supportive care needs over time and its associated factors, the outcome of interest which is the level of needs of the subject for all five domains will be measured continuously by a total domain score. A two-way repeated-measures multivariate analysis of variance will be used to determine differences in multiple needs in all five domains over time or between-subjects characteristics. Any associations between sociodemographic characteristics (age, gender, ethnicity, marital status and family history of breast cancer); clinical status (diabetes, hypertension and heart disease); treatment history (chemotherapy, radiotherapy, surgery or hormone therapy); coping behaviour; social support; and PTG, with changes in supportive care needs among patients with breast cancer, will be assessed. The level of significance will be set at 0.05 with a $95 \%$ CI. 


\section{Data and study tools}

Data will be collected using six tools: the medical records; pro-forma data collection; the 34-item Short-Form Supportive Care Needs Survey-Malay version (SCNSSF34-M); the brief Coping Orientation to Problems Experienced-Malay version (COPE-M); Source of Social Support Scale-Malay version (SSSS-M) and the Malay PTG Inventory-Short Form (PTGI-SF-M).

\section{Patients' medical records}

Patients' medical records are required for information including patients' sociodemographic characteristics, telephone number, date of breast cancer diagnosis, next follow-up, treatment-related factors, clinical background, cancer subtypes and pathological characteristics. The sociodemographic factors assessed in this study include patients' age, gender, ethnicity, marital status and family history of breast cancer. The clinical background assesses on the status of other medical comorbidities (diabetes, hypertension and heart disease). The cancer subtypes identify patients based on oestrogen receptor, progesterone receptor, HER2 or triple-negative. The treatmentrelated factors to be assessed include the type of breast cancer treatment received between the breast cancer diagnosis and the end 6-month treatment. This includes any/combination of chemotherapy, radiotherapy, surgery or hormonal therapy. The start date of each treatment will be recorded. Pathological characteristics include tumour size, staging, metastasis status and lymph nodes involvement.

\section{Data collection pro forma}

All information gathered from patients' medical records will be filled in a data collection form by the appointed research assistant and the second author (NA).

\section{Supportive care needs}

Data on patients' supportive care needs will be assessed using the SCNS-SF34-M. The original English version of this survey questionnaire has been validated and has shown high internal consistency with Cronbach's alpha coefficients ranging from 0.86 to $0.96{ }^{5333} \mathrm{~A}$ total of 34 items are divided into five domains:

1. Psychological (10 items) -item numbers 6-14 and 17

2. PDL ( 5 items) -item numbers $1-5$

3. Sexuality (3 items)-item numbers 15, 16 and 31

4. Health system and information (11 items)-item numbers 23-30; 32-34

5. Patient care and support (5 items)-item numbers 18-22

For each item, the respondents will be required to indicate their level of needs over the past month concerning having breast cancer. The level of needs would be scored on a 5-point Likert scale: (1) not applicable, (2) satisfied, (3) low need, (4) moderate need, (5) high need. The domain score will be obtained by adding together the responses to each of the items within the domain and dividing the sum by the number of items in the domain according to the scoring manual. ${ }^{44}$ The score has possible values ranging from 0 to 100 , with a higher score indicating more unmet needs.

The Malay version of this SCNS-SF34 will be prepared for those who cannot speak English. Translation into the Malay language will be done by an independent language expert who is fluent in both English and Malay languages. Back-to-back translation (Malay-English-Malay) will be carried out by independent language experts not associated with this study. Later, both of the two sets of English and Malay versions will be subsequently compared with the original version by breast and reconstructive surgeons for the conceptual equivalence of the items (face validity). Changes to the translated version will be made as needed to remove ambiguity and will be pretested before the real study.

\section{Coping behaviour}

The coping behaviour of the patients will be assessed using the validated brief COPE-M scale. ${ }^{54}$ The system uses a 4-point Likert scale and comprises 28 items, ranging from 'I have not been doing this at all' (1) to 'I have been doing this a lot' (4). The higher score represents greater coping strategies used by the patients. In total, 14 dimensions (two items for every dimension) are put forward by this scale. These are self-distraction, active coping, denial, substance use, use of emotional support, use of instrumental support, behavioural disengagement, venting, positive reframing, planning, humour, acceptance, religion and self-blame. ${ }^{54}$

\section{Social support and PTG}

This study will assess the patients' sources of social support and the PTG experienced following a breast cancer diagnosis.

\section{Source of social support}

The patients' source of social support will be measured by using the SSSS-M. The translated Malay version was adapted from the original SSSS used among patients with cancer by Kinsinger et al. It has been previously validated for use with patients with local breast cancer. ${ }^{556}$ It comprises a 10-item respondent-rated questionnaire that is used to measure the degree of spousal social support perceived by the respondent. It consists of four domains: informational support (measures the degree to which the spouse of the patient provides guidance, advice and feedback about the patient's problems); instrumental support (measures the degree to which the spouse of the patient assists in managing the patient's daily chores, such as transportation, financial management and household chores); emotional support (assesses the degree to which the spouse of the patient listens, empathises, communicates sufficiently and provides comfort to the patient); and negative support (measures the degree to which the spouse of the patient neglects the supportive needs of the patient). The SSSS-M has been validated in patients with breast cancer with confirmatory factor analysis and had good psychometric properties. 


\section{Malay PTG Inventory-Short Form}

The patients' PTG will be measured using the PTGI-SF-M. The translated Malay version has been adapted from the original PTGI-SF developed by Cann et al and has been validated previously among patients with local breast cancer. ${ }^{57} 58$ It comprises a 10 -item respondent-rated questionnaire used to measure five domains: the appreciation of life, personal strength, relating to others, spiritual growth and new possibilities in life. Each item is rated from 0 (I did not experience this change) to 5 (I experienced this change to a great degree). It is scored from 0 to 50 , and the higher the total score, the higher the level of PTG. ${ }^{58}$

\section{Patient and public involvement}

The patients in this study will not be involved in developing the research question or study design. They will also not be involved in the recruitment or conduct of the study.

\section{Outcomes}

This study's primary outcome is the changes in supportive care needs among patients with breast cancer over three time points across their cancer treatment trajectory. This study's secondary outcome is to determine associations between patients' characteristics, clinical treatment history, coping behaviour, social support and PTG to the changes in unmet supportive care needs over time.

\section{ETHICS AND DISSEMINATION}

\section{Dissemination and data sharing}

This study has been registered with the Malaysian National Medical Research Registry and has obtained ethical approval for the observational study from two established human ethics committees: the Medical Research and Ethics Committee, Ministry of Health Malaysia (NMRR-19-268-45809 IIR) and the Human Research Ethics Committee of Universiti Sains Malaysia (USM/JEPeM/17100443). The researchers will adhere to the Declaration of Helsinki principles and the Malaysian Good Clinical Practice Guidelines throughout the study. This study will be reported following the Strengthening the Reporting of Observational Studies in Epidemiology Statement: Guidelines for Reporting Observational Studies to enhance reporting transparency. ${ }^{59}$ Data and resources will be shared with other eligible investigators through academically established means. The datasets used and analysed during the study will be available from the corresponding author upon reasonable request. All aspects of the study, including results, will be strictly confidential, and only the study team will have access to information on participants, except as required by law. In publication and presentation of the results, no reference will be made to individual subjects in a form that will expose their identity. A report of the study may be submitted for publication. However, individual participants will not be identifiable in such a report. Data will be presented as grouped data and will not individually identify the respondents.

\section{Data storage and management}

All data will be entered by the principal investigators (PIs) or research assistants, and data accuracy will be verified by the study PI. Data quality control measures will include queries to identify missing data, outliers and discrepancies. Only research assistants and PIs will have access to protected health information. After enrolment, a unique identifier will be assigned to each study subject. The data from all sites will be uploaded and stored in a passwordencrypted external hard disk which will only be accessible to the PI. All computers used in this study will be password protected and encrypted per university policy to increase security. The PI will ensure that the anonymity is maintained. Patients will not be identified by name in any reports on this study. The study PI will have access to the final study dataset. All data collection forms and questionnaires will be maintained in lockable filing cabinets within the corresponding author's office. All data will be kept for 7 years. To reidentify data at a later stage for feedback to participants, the data will be stored in the identifiable or potentially identifiable (coded) form. After the prescribed storage time, all hard paper copies will be shredded before placement in a secure paper recycling bin. All soft copy data will be deleted.

\section{Strengths and limitations \\ Strengths}

Our longitudinal study design, in which parallel covariates are reliably and repeatedly measured over time, will allow us to look at changes of supportive care needs over time in the same patient, defining the temporal sequence of changes and providing stronger evidence for a wide range of longitudinal data. The PenBCNeeds Study should provide evidence for incidence and risk factors of the unmet supportive care needs among newly diagnosed patients with breast cancer, benefiting numerous healthcare stakeholders and assisting in the design of a better cancer supportive treatment in future.

\section{Limitations}

This study may experience a low response rate, loss to follow-up in the second and third telephone interviews, and missing data points that would challenge the internal validity of reported results from the PenBCNeeds Study. To minimise the issue of low response rate and loss to follow-up among the eligible patients, a strong rapport will be emphasised between the research assistant and each patient during the first interview. This entails respecting the time commitment of patients, formal tracking of patients (including acquiring multiple contacts for arranging follow-up), strong interpersonal skills among study personnel and flexible hours for telephone interviews.

Acknowledgements The authors thank those who gave invaluable comments on drafts of this protocol. 
Contributors NMS - conception and study design, acquisition of data, analysis and interpretation of data, drafting and revising the manuscript. NA—conception and study design, acquisition of data, analysis and interpretation of data, drafting and revising the manuscript. $\mathrm{RH}$ - study design, acquisition of data, analysis and interpretation of data, drafting and revising the manuscript. NMMM — study design, acquisition of data, analysis and interpretation of data, drafting and revising the manuscript. MFILBA — study design, acquisition of data, analysis and interpretation of data, drafting and revising the manuscript. All authors have read and given final approval for the submitted manuscript.

Funding This work was supported by the Universiti Sains Malaysia Short Term Grant (grant number: 304.CIPPT.6315102).

\section{Competing interests None declared.}

Patient and public involvement Patients and/or the public were not involved in the design, or conduct, or reporting, or dissemination plans of this research.

Patient consent for publication Not required.

Provenance and peer review Not commissioned; externally peer reviewed.

Open access This is an open access article distributed in accordance with the Creative Commons Attribution Non Commercial (CC BY-NC 4.0) license, which permits others to distribute, remix, adapt, build upon this work non-commercially, and license their derivative works on different terms, provided the original work is properly cited, appropriate credit is given, any changes made indicated, and the use is non-commercial. See: http://creativecommons.org/licenses/by-nc/4.0/.

ORCID iDs

Noorsuzana Mohd Shariff http://orcid.org/0000-0002-1692-8626

Mohammad Farris Iman Leong Bin Abdullah http://orcid.org/0000-0002-7762-4052

\section{REFERENCES}

1 Globocan Observatory 2019 WI, (IARC) International Agency for Reseach on Cancer, (WHO) WHO. Breast cancer. source: Globocan 2018. World Heal Organ Int Agency Res Cancer 2019;876:2018-9 http://gco.iarc.fr/today\%0Ahttps://gco.iarc.fr/today/data/factsheets/ populations $/ 900$-world-fact-sheets.pdf

2 Azizah AM, Hashimah B, Nirmal K. Malaysia National cancer registry report (MNCR) 2019:2012-6.

3 Shi Q, Smith TG, Michonski JD, et al. Symptom burden in cancer survivors 1 year after diagnosis. Cancer 2011;117:2779-90.

4 Fong EJ, Cheah WL. Unmet supportive care needs among breast cancer survivors of community-based support group in Kuching, Sarawak. Int J Breast Cancer 2016;2016:1-9.

5 Binkley JM, Harris SR, Levangie PK, et al. Patient perspectives on breast cancer treatment side effects and the prospective surveillance model for physical rehabilitation for women with breast cancer. Cancer 2012;118:2207-16.

6 Ahmad S, Fergus K, McCarthy M. Psychosocial issues experienced by young women with breast cancer: the minority group with the majority of need. Curr Opin Support Palliat Care 2015;9:271-8.

7 Scotté F. The importance of supportive care in optimizing treatment outcomes of patients with advanced prostate cancer. Oncologist 2012;17 Suppl 1:23-30.

8 Mohammadzadeh Nimekari M, Saei Ghare Naz M, Ashouri Taziani $\mathrm{Y}$, et al. Correlation between supportive care needs of women with breast cancer and quality of life of their family caregivers. Int $J$ Community Based Nurs Midwifery 2019;7:300-8.

9 Carter N, Bryant-Lukosius D, DiCenso A, et al. The supportive care needs of men with advanced prostate cancer. Oncol Nurs Forum 2011;38:189-98.

10 Edib Z, Kumarasamy V, binti Abdullah N. Most prevalent unmet supportive care needs and quality of life of breast cancer patients in a tertiary hospital in Malaysia. Health Qual Life Outcomes 2016;14:1-10.

11 Carr W, Wolfe S. Unmet needs as sociomedical indicators. Int $J$ Health Serv 1976;6:417-30.

12 Vetsch J, Fardell JE, Wakefield CE, et al. "Forewarned and forearmed": Long-term childhood cancer survivors' and parents' information needs and implications for survivorship models of care. Patient Educ Couns 2017;100:355-63.

13 Griesser A-C, Vlastos G, Morel L, et al. Socio-Demographic predictors of high support needs in newly diagnosed breast cancer patients. Eur J Cancer Care 2011;20:466-74.

14 Capelan M, Battisti NML, McLoughlin A, et al. The prevalence of unmet needs in 625 women living beyond a diagnosis of early breast cancer. Br J Cancer 2017;117:1113-20.
15 Cheng KKF, Cheng HL, Wong WH, et al. A mixed-methods study to explore the supportive care needs of breast cancer survivors. Psychooncology 2018;27:265-71.

16 Kemp E, Koczwara B, Butow P, et al. Online information and support needs of women with advanced breast cancer: a qualitative analysis. Support Care Cancer 2018;26:3489-96.

17 Klungrit S, Thanasilp S, Jitpanya C. Supportive care needs: an aspect of Thai women with breast cancer undergoing chemotherapy. Eur J Oncol Nurs 2019;41:82-7.

18 Chae BJ, Lee J, Lee SK, et al. Unmet needs and related factors of Korean breast cancer survivors: a multicenter, cross-sectional study. BMC Cancer 2019;19:839.

19 Chou Y-H, Chia-Rong Hsieh V, Chen X, et al. Unmet supportive care needs of survival patients with breast cancer in different cancer stages and treatment phases. Taiwan J Obstet Gynecol 2020;59:231-6.

20 Pérez-Fortis A, Fleer J, Schroevers MJ, et al. Course and predictors of supportive care needs among Mexican breast cancer patients: a longitudinal study. Psychooncology 2018;27:2132-40.

21 Schmid-Büchi S, Halfens RJG, Müller M, et al. Factors associated with supportive care needs of patients under treatment for breast cancer. Eur J Oncol Nurs 2013;17:22-9.

22 Martínez Arroyo O, Andreu Vaíllo Y, Martínez López P, et al. Emotional distress and unmet supportive care needs in survivors of breast cancer beyond the end of primary treatment. Support Care Cancer 2019;27:1049-57.

23 Fiszer C, Dolbeault S, Sultan S, et al. Prevalence, intensity, and predictors of the supportive care needs of women diagnosed with breast cancer: a systematic review. Psychooncology 2014;23:361-74.

24 Au A, Lam W, Tsang J, et al. Supportive care needs in Hong Kong Chinese women confronting advanced breast cancer. Psychooncology 2013;22:1144-51.

25 von Heymann-Horan AB, Dalton SO, Dziekanska A, et al. Unmet needs of women with breast cancer during and after primary treatment: a prospective study in Denmark. Acta Oncol 2013;52:382-90.

26 Qualheim RE, Gall EA. Breast carcinoma with multiple sites of origin. Cancer 1957;10:460-8.

27 Chu J, Bae H, Seo Y, et al. The prognostic impact of synchronous ipsilateral multiple breast cancer: survival outcomes according to the eighth American joint Committee on cancer staging and molecular subtype. J Pathol Transl Med 2018;52:396-403.

28 Liao M-N, Chen S-C, Chen S-C, et al. Changes and predictors of unmet supportive care needs in Taiwanese women with newly diagnosed breast cancer. Oncol Nurs Forum 2012;39:E380-9.

29 Burris JL, Armeson K, Sterba KR. A closer look at unmet needs at the end of primary treatment for breast cancer: a longitudinal pilot study. Behav Med 2015;41:69-76.

30 Pérez-Fortis A, Fleer J, Sánchez-Sosa JJ, et al. Prevalence and factors associated with supportive care needs among newly diagnosed Mexican breast cancer patients. Support Care Cancer 2017;25:3273-80.

31 Lam WWT, Tsang J, Yeo W, et al. The evolution of supportive care needs trajectories in women with advanced breast cancer during the 12 months following diagnosis. Support Care Cancer 2014;22:635-44.

32 Cardoso F, Bese N, Distelhorst SR, et al. Supportive care during treatment for breast cancer: resource allocations in low- and middleincome countries. A breast health global initiative 2013 consensus statement. Breast 2013;22:593-605.

33 Weißflog G, Hönig K, Gündel H, et al. Associations between dyadic coping and supportive care needs: findings from a study with hematologic cancer patients and their partners. Support Care Cancer 2017;25:1445-54.

34 Lin N, Simeone RS, Ensel WM, et al. Social support, stressful life events, and illness: a model and an empirical test. $J$ Health Soc Behav 1979;20:108-19.

35 Shand LK, Cowlishaw S, Brooker JE, et al. Correlates of posttraumatic stress symptoms and growth in cancer patients: a systematic review and meta-analysis. Psychooncology 2015;24:624-34.

36 Williams GR, Pisu M, Rocque GB, et al. Unmet social support needs among older adults with cancer. Cancer 2019;125:473-81.

37 Tedeschi RG, Calhoun LG. TARGET ARTICLE: "Posttraumatic Growth: Conceptual Foundations and Empirical Evidence". Psychol Inq 2004;15:1-18.

38 Casellas-Grau A, Ochoa C, Ruini C. Psychological and clinical correlates of posttraumatic growth in cancer: a systematic and critical review. Psychooncology 2017;26:2007-18. 
39 Leong Abdullah MFI, Hami R, Appalanaido GK, et al. Diagnosis of cancer is not a death sentence: examining posttraumatic growth and its associated factors in cancer patients. J Psychosoc Oncol 2019;37:636-51.

40 Arifin WN. Introduction to sample size calculation. Educ Med J 2013;5:89-96.

41 Etikan I. Comparison of convenience sampling and Purposive sampling. Am J Theor App/ Stat 2016;5:1.

42 Newell S, Sanson-Fisher RW, Girgis A, et al. How well do medical oncologists' perceptions reflect their patients' reported physical and psychosocial problems? data from a survey of five oncologists. Cancer 1998;83:1640-51.

43 Rainbird K, Perkins J, Sanson-Fisher R, et al. The needs of patients with advanced, incurable cancer. Br J Cancer 2009;101:759-64.

44 Macleduff P, Boyes A, Zucca A. Supportive Care Needs Survey : A guide to administration, scoring and analysis. Aust Univ New Castle 2004.

45 Wang S, Li Y, Li C, et al. Distribution and determinants of unmet need for supportive care among women with breast cancer in China. Med Sci Monit 2018;24:1680-7.

46 Bowling A. Mode of questionnaire administration can have serious effects on data quality. J Public Health 2005;27:281-91.

47 Sarantakos S. Social research. 2nd edn. Australia: Macmillan, 1998.

48 Fowler FJ. Survey research methods. Newbury Park, CA: Sage, 2001.

49 Dillman DA, Gallegos JG, Frey JH. Reducing refusal rates for telephone interviews. Public Opinion Quarterly 1976;40:66-78.

50 Marcus AC, Crane LA. Telephone surveys in public health research. Med Care 1986;24:97-112.
51 Taylor A. I'll call you back on my mobile: A critique of the telephone interview with adolescent boys. Westminster Studies in Education 2002;5:19-34.

52 Block ES, Erskine L. Interviewing by telephone: specific considerations, opportunities, and challenges. Int J Qual Methods 2012;11:428-45.

53 Boyes A, Girgis A, Lecathelinais C. Brief assessment of adult cancer patients' perceived needs: development and validation of the 34item supportive care needs survey (SCNS-SF34). J Eval Clin Pract 2009;15:602-6.

54 Yusoff N, Low WY, Yip CH. Cope Scale : a Study on Malaysian Women Treated With. MPJ Online Early 2009:1-9.

55 Kinsinger SW, Laurenceau J-P, Carver CS, et al. Perceived partner support and psychosexual adjustment to breast cancer. Psychol Health 2011;26:1571-88.

56 Abdullah M, Hami R, Azman N. Validation of the Malay version of the sources of social support scale among Malaysian cancer patients. ASEAN J Psychiatry 2017;18:0

57 Cann A, Calhoun LG, Tedeschi RG. A short form of the posttraumatic growth inventory. Anxiety, Stress Coping 2010:23:127-37.

58 Abdullah M, Hami R, Appalanaido GK. Validation of the Malay version of the posttraumatic growth inventory-short form (PTGI-SF) among Malaysian cancer patients. ASEAN J Psychiatry 2017;18:0

59 Von Elm E, Altman DG, Egger M. The strengthening the reporting of observational studies in epidemiology (STROBE) statement: guidelines for reporting observational studies. UroToday Int $J$ 2009;2:20-2. 